\title{
Strong Field Atomic Physics in the Mid-Infrared
}

B. Sheehy, J. D. D. Martin, T. O. Clatterbuck, D. W. Kim, L. F. DiMauro, P. Agostini, K. J. Schafer, M. B. Gaarde, K. C. Kulander

This article was submitted to

Multiphoton Processes 1999: $8^{\text {th }}$ International Conference on Multiphoton Processes, Monterey, CA,, October 3-8, 1999

\section{March 15, 2000}

U.S. Department of Energy

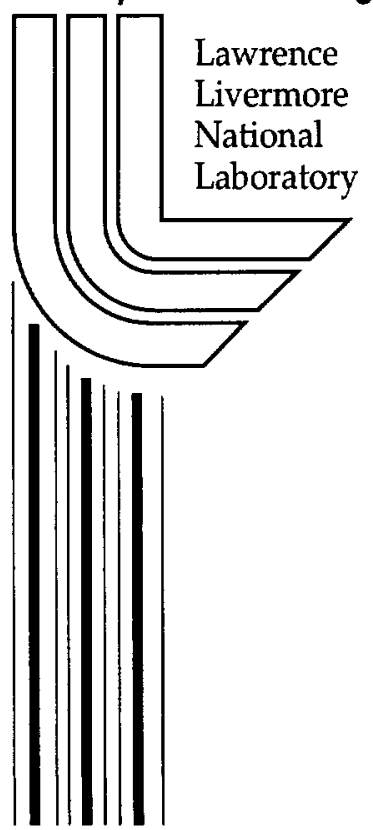




\section{DISCLAIMER}

This document was prepared as an account of work sponsored by an agency of the United States Government. Neither the United States Government nor the University of California nor any of their employees, makes any warranty, express or implied, or assumes any legal liability or responsibility for the accuracy, completeness, or usefulness of any information, apparatus, product, or process disclosed, or represents that its use would not infringe privately owned rights. Reference herein to any specific commercial product, process, or service by trade name, trademark, manufacturer, or otherwise, does not necessarily constitute or imply its endorsement, recommendation, or favoring by the United States Government or the University of California. The views and opinions of authors expressed herein do not necessarily state or reflect those of the United States Government or the University of California, and shall not be used for advertising or product endorsement purposes.

This is a preprint of a paper intended for publication in a journal or proceedings. Since changes may be made before publication, this preprint is made available with the understanding that it will not be cited or reproduced without the permission of the author.

This work was performed under the auspices of the United States Department of Energy by the University of California, Lawrence Livermore National Laboratory under contract No. W-7405-Eng-48.

This report has been reproduced directly from the best available copy.

Available electronically at http://www.doc.gov/bridge

Available for a processing fee to U.S. Department of Energy

And its contractors in paper from

U.S. Department of Energy

Office of Scientific and Technical Information

P.O. Box 62

Oak Ridge, TN 37831-0062

Telephone: (865) 576-8401

Facsimile: (865) 576-5728

E-mail: reports@adonis.osti.gov

Available for the sale to the public from

U.S. Department of Commerce

National Technical Information Service

5285 Port Royal Road

Springfield, VA 22161

Telephone: (800) 553-6847

Facsimile: (703) 605-6900

E-mail: orders@ntis.fedworld.gov

Online ordering: $\underline{\text { http://www.ntis.gov/ordering.htm }}$

OR

Lawrence Livermore National Laboratory

Technical Information Department's Digital Library

http://www.llnl.gov/tid/Library.html 


\title{
Strong Field Atomic Physics In The Mid- Infrared
}

\author{
B. Sheehy ${ }^{1}$, J. D. D. Martin ${ }^{1}$, T. O. Clatterbuck ${ }^{1}$, D. W. Kim ${ }^{1,2}$, L.F. \\ DiMauro $^{1}$, P. Agostini ${ }^{3}$, K. J. Schafer ${ }^{4}$. M. B. Gaarde ${ }^{4,5}$, and K. C. \\ Kulander $^{6}$ \\ ${ }^{1}$ Chemistry Department, Brookhaven National Laboratory, Upton, New York 11973. \\ ${ }^{2}$ Research Institute of Industrial Science \& Technology, Pohang, Korea 790-300 \\ ${ }^{3}$ SPAM, Centre d'Etudes de Sacloy, 91191 Gif Sur Yvette, France. \\ ${ }^{4}$ Department of Physics and Astronomy, Louisiana State University, Baton Rouge, Louisiana 70803. \\ SLund Institute Technology, P.O. Box 118, S-22100 Lund, Sweden. \\ ${ }^{6}$ TAMP, Lawrence Livermore National Laboratory, Livermore, California 94551
}

\begin{abstract}
We examine strong field atomic physics in a wavelength region (3-4 microns) where very little work has previously been done. The soft photon energy allows the exploration of oneelectron atoms with low binding energies (alkali metals). We find that photoionization spectra differ from rare gas studies at shorter wavelengths due to more complex ion core potentials. Harmonic generation is studied, and we find that harmonic bandwidths are consistent with theory and the possibility of compression to pulse widths much shorter than that of the driving pulse. Harmonic yields in the visible and UV are sufficient for a complete study of their amplitude and phase characteristics.
\end{abstract}

\section{INTRODUCTION}

The interaction of atoms with strong optical fields has given rise to many new phenomena that have been as interesting in their fundamental physics as they are rich in potential applications. In recent years, our understanding has progressed so that many diverse phenomena may be modeled within a simple theoretical framework, while short-pulse technology has matured so that applications are more promising than ever. A great deal of the experimental work has been done in the near-infrared, visible, and UV parts of the spectrum, as this is where the development of short-pulse technology has afforded the most intense pulses. However, there are reasons to expect both interesting differences in the fundamental physics and more insight into applications by working at longer wavelengths. In this paper we explore some of these differences both theoretically and experimentally.

Many strong field phenomena may be understood in terms of the "rescattering model" [1-3], in which an electron is first promoted into the continuum by a tunneling process, and subsequently accelerated in the optical field. The electron's motion in the optical field may return it to the vicinity of the ion core, where it may radiatively recombine or scatter. This model has been very successful in predicting single- 
electron effects, including many features of high harmonic generation (HHG) $[2,3]$ and photoelectron energy spectra (PES) in above-threshold ionization (ATI) $[4,5]$. Extending experimental studies of these phenomena to longer wavelengths is appealing because the characteristic parameters in the rescattering model all scale differently with the wavelength of the light. The tunneling-ionization step may be parameterized by the Keldysh adiabaticity parameter $\gamma[5]$, which scales as $1 / \lambda$. This dimensionless parameter is given by $\gamma=\sqrt{I_{p} / 2 U_{p}}$, where $I_{p}$ is the atomic ionization potential and $U_{p}$, the ponderomotive potential, is the cycle-averaged kinetic energy of a free electron moving in the optical field. The Keldysh parameter compares the characteristic time $\tau$ for tunneling through the field-suppressed Coulomb barrier to the optical period. Smaller ionization potentials or larger intensities decrease $\tau$, while longer wavelengths increase the optical period, decreasing $\gamma$ and improving the tunneling approximation. For values of $\gamma$ much less than 1 , tunneling ionization dominates. In comparing different atoms irradiated by different fields, it is instructive to compare experiments in which the values of $\gamma$ are similar, as the physics of the ionization step is comparable. Thus, experiments on the alkalis in the mid-infrared are comparable to rare gas experiments in the near-infrared at much larger intensities. For example, at $3.5 \mu \mathrm{m}, \gamma$ equals 1 at an intensity of $1.9 \mathrm{TW} / \mathrm{cm}^{2}$ for potassium, while for argon in a $0.8 \mu \mathrm{m}$ field, $\gamma$ equals 1 at $130 \mathrm{TW} / \mathrm{cm}^{2}$.

While $\gamma$ scales as $1 / \lambda$, the second dynamical step in the rescattering model, the energy acquired by the electron as it is accelerated in the optical field, scales as $\lambda^{2}$. At equal intensities, electron energies are an order of magnitude larger in the mid-IR than in the near-IR. Yet another scaling enters the problem when one considers the time between the tunneling ionization step and the return of the electron to the ion core. This time scales linearly with $\lambda$ and is important because the electron wave packet spreads during this time, reducing its overlap with the core and the probability of scattering or recombining.

Other differences are also of interest. Consider the coupling of excited states in the argon/potassium comparison above. In argon at $0.8 \mu \mathrm{m}$, the first excited state lies 8 photons above the ground state, while only 11 photons are required to reach the ionization threshold. The ionization of potassium at $3.5 \mu \mathrm{m}$ is of slightly higher order (13 photons), but the first excited state lies just 5 photons above the ground state. The role of this coupling is further explored in another paper in this volume [6]. The variation between alkali species in the ionic core potential also provides a good detailed test of the rescattering model. As shown below, small differences in the core potential can manifest themselves quite clearly in the PES by their effect on the rescattering process.

One of the most promising applications of strong-field physics is the production of short wavelength radiation through HHG. Harmonic orders as high as the $401^{\text {st }}$ have been reached, producing wavelengths as short as $2 \mathrm{~nm}$ [7]. Recent theoretical studies $[8,9]$ indicate that it is likely that the coherence properties of the high harmonics may be exploited in order to generate pulses on an attosecond time scale. Experimentally, it is difficult to test these theories at such short wavelengths. By using a fundamental wavelength in the mid-IR, harmonics may be generated in the visible and ultraviolet, 
where their coherence properties may be more easily measured. We have developed a mid-infrared source of sufficient intensity and produced visible harmonics at levels adequate to do these measurements. We have measured the spectral properties of some of these harmonics, and compared their relative abundance with theoretical predictions. We find that the spectral measurements are consistent with dramatic pulse shortening. Conclusive evidence will require the temporal and coherence measurements that are underway.

\section{MID-INFRARED SOURCE}

Tunable mid-IR light is generated by three-wave mixing in a potassium titanyl phosphate (KTP) crystal. A block diagram of the laser system is shown in Fig. 1. The tunable pump source includes a Spectra Physics Tsunami Ti:Sapphire mode-locked oscillator, pumped by a diode-pumped, intracavity doubled neodymium vanadate laser (Spectra Physics Millennia). The $1.5 \mathrm{psec}$ pulses are stretched to $100 \mathrm{psec}$ using a 2000 groove/mm grating in a stretcher of standard all-reflective design. A regenerative Ti:Sapphire amplifier, pumped by $12 \mathrm{~mJ}, 527 \mathrm{~nm}, 170 \mathrm{nsec}$ pulses from a Quantronix model 527 Q-switched, intracavity-doubled, Nd:YLF laser, picks pulses from the 80 $\mathrm{MHz}$ oscillator train at $1 \mathrm{kHz}$ and amplifies them to $2.8 \mathrm{~mJ}$. After losses in the compressor, $1.6 \mathrm{~mJ}$ of radiation tunable from $780-830 \mathrm{~nm}$ is available for mid-IR production.

The signal beam is fixed in frequency at $1053 \mathrm{~nm}$. A diode pumped mode-locked Nd:YLF laser (Lightwave model 131) produces $25 \mathrm{psec}, 2.5 \mathrm{~nJ}$ pulses at $80 \mathrm{MHz}$. These are picked at $1 \mathrm{kHz}$ and amplified to a maximum energy of $1.8 \mathrm{~mJ}$ in a discharge lamp pumped regenerative amplifier built in our laboratory. The parametric process requires the temporal overlap of the pulses in the mixing crystal. The synchronization requirement is somewhat relaxed by using a signal seed pulse that is much longer than the pump pulse [10]. As the amplifiers introduce no additional jitter, the problem reduces to one of synchronizing the oscillators to much less than the signal pulse width. Both oscillators are locked to the same RF reference using a phaselocked loop that feeds back to the cavity lengths. The two oscillators were crosscorrelated using sum-frequency generation in $\beta$-Barium borate, and the jitter found to be less than 5 psec (the resolution limit of the apparatus).

In all of the experiments described here, the three-wave mixing was done using type-II phase matching in a $5 \mathrm{~mm}$ long KTP crystal cut in the X-Z plane at 40 degrees. Potassium titanyl arsenate (KTA) and Potassium niobate $\left(\mathrm{KNbO}_{3}\right)$ have also been used, with comparable conversion efficiency. At shorter pulse widths and longer wavelengths, the group velocity mismatch and transmission curves of the crystals dictates the best crystal choice. The pump and signal beams are focussed to $0.9 \mathrm{~mm}$ and $1.5 \mathrm{~mm}$ diameter spots respectively, with a 1 degree angle between them in the direction perpendicular to the phase-matching plane. The small angle facilitates the separation of the idler beam: the centers of the pump and signal beam are blocked 20 $\mathrm{cm}$ downstream, and the residual pump and signal light is filtered out by collimating the idler with a silicon lens. 
The system produces $100 \mu \mathrm{J}, 1.5$ psec pulses at $1 \mathrm{kHz}$, tunable between 3 and 4.1 microns, from inputs of $1.6 \mathrm{~mJ}$ in the pump beam and $0.3 \mathrm{~mJ}$ in the signal beam. The signal beam acts only as a seed, and is saturated at this level. The system's tunability can be extended to cover $2.5-5$ microns with minor optics changes in the oscillator and regenerative amplifier. The Tsunami oscillator can also be easily configured to run at $100 \mathrm{fsec}$ pulse widths. With a grating change in the stretcher/compressor, the system may then be used to produce $100 \mathrm{fsec}$ pulses in the mid-IR.

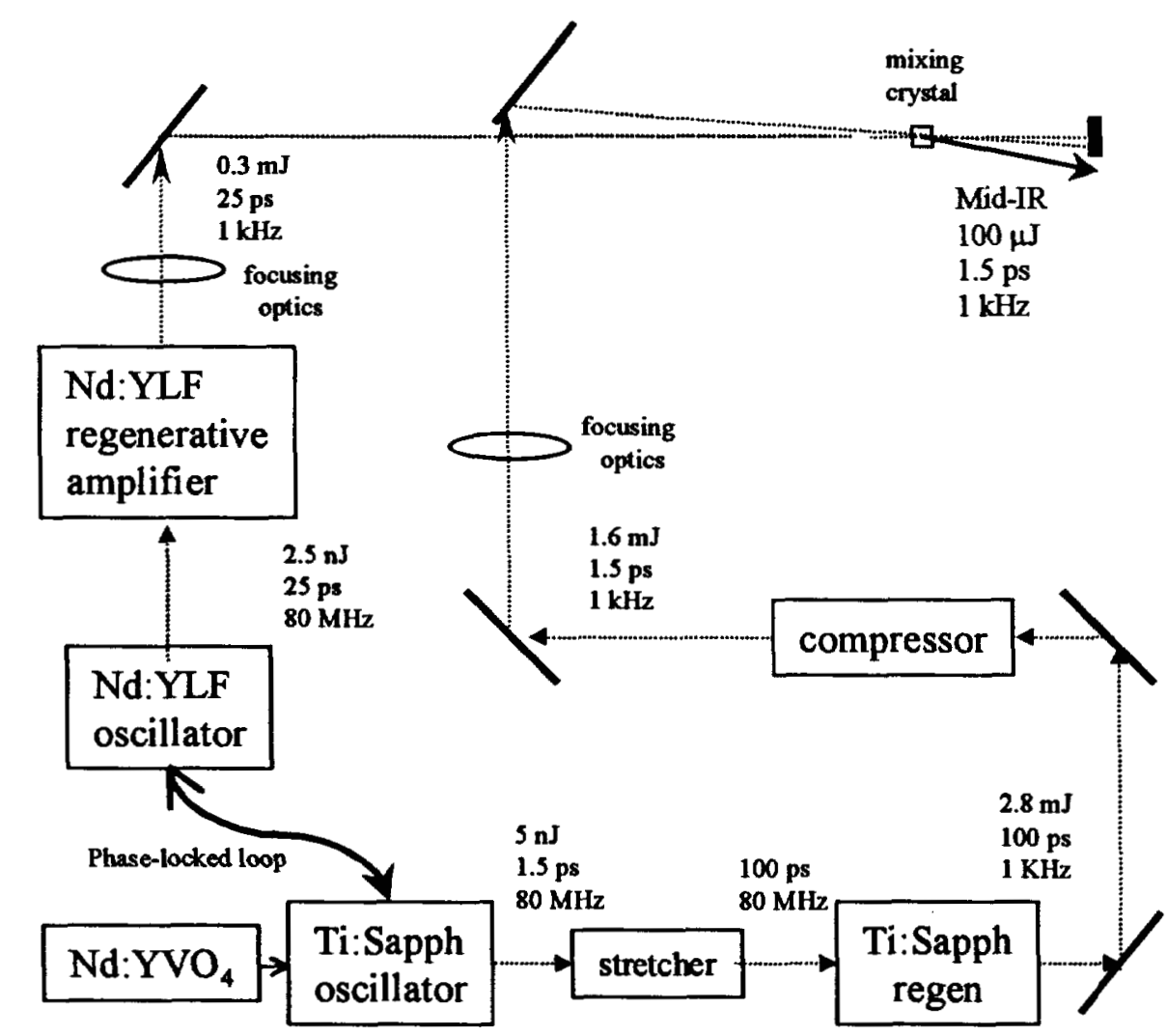

FIGURE 1. Block diagram of the mid-infrared source.

\section{PHOTOIONIZATION EXPERIMENTS}

The rescattering model's predictions about the gross structure of high harmonic and electron spectra follow from very simple considerations of an electron's motion in an oscillating field. An electron that never revisits the ion core can acquire a maximum drift energy of $2 U_{p}$, if it does return, its maximum return energy is $3.2 U_{p}$, while if it elastically scatters and is accelerated again in the field, the maximum drift energy that it can attain is $10 U_{p}$. The well known $3 U_{p}+I_{p}$ cutoff in high harmonic spectra occurs 
at the maximum return energy [11]. Photoelectron spectra of helium and neon in the tunneling regime show a dramatic change at $2 \mathrm{U}_{\mathrm{p}}$, and the plateau shape observed in the high energy tail is given by modeling the rescattering of the returning electron wave packet with the parent ion [4,5]. In the case of the rare gases, the scattering potentials presented by the ion cores of the different atomic species do not differ enough to appreciably change the shape of the high-energy part $\left(>2 U_{p}\right)$ of the PES. In the alkali atoms there is more variation.

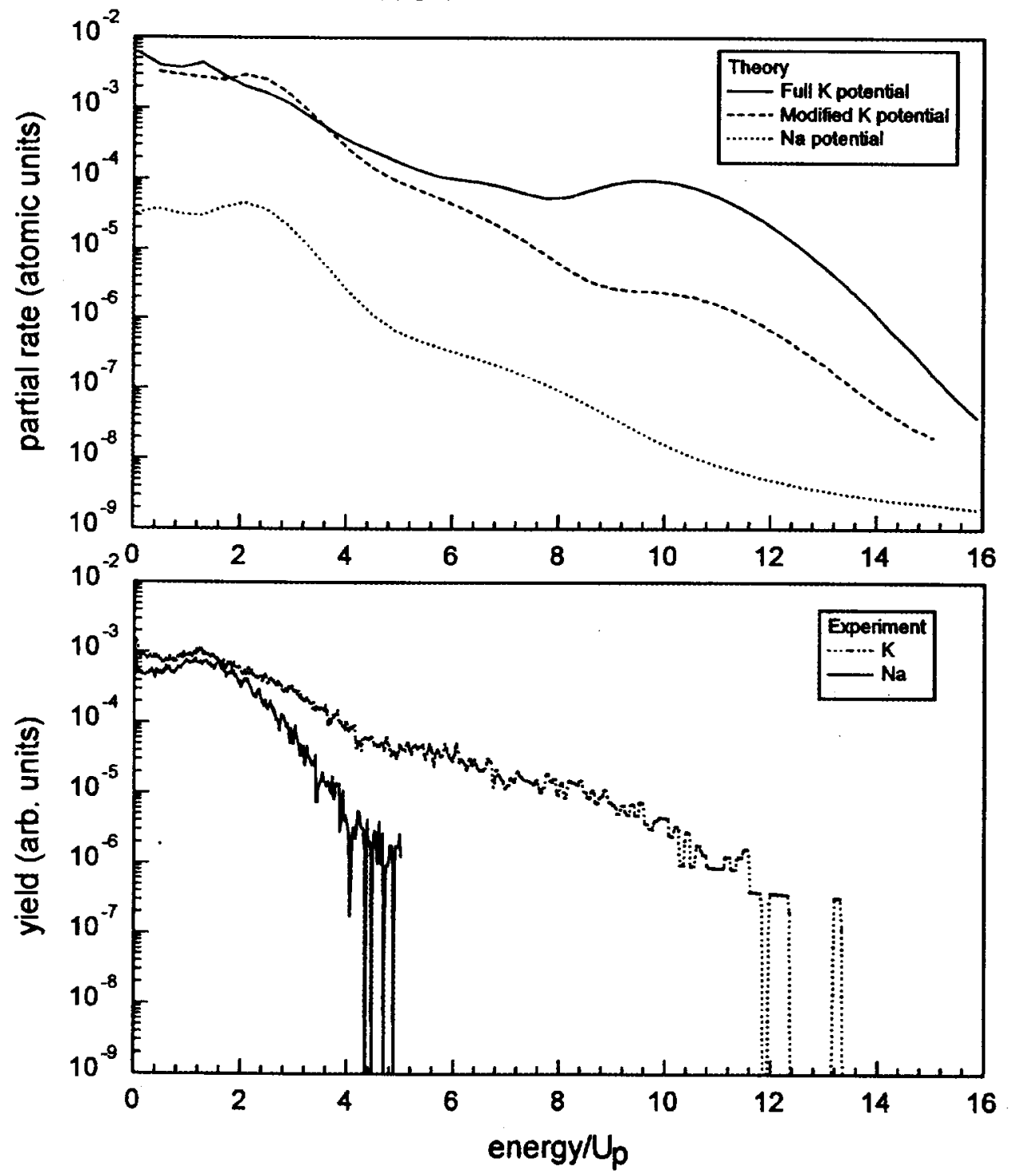

FIGURE 2. Theoretical and experimental photoelectron spectra for sodium and potassium excited by $3.2 \mu \mathrm{m}$ radiation. The theoretical curves are calculated for a peak intensity of $1 \mathrm{TW} / \mathrm{cm}^{2}$. The modified $\mathrm{K}$ potential is formed by replacing the potassium $d$ potential with that of hydrogen. The $\mathrm{K}$ an $\mathrm{Na}$ experimental spectra are taken at $1.4(5) \mathrm{TW} / \mathrm{cm}^{2}$ and $1.7(6) \mathrm{TW} / \mathrm{cm}^{2}$ respectively. The experimental curves have been shifted on the y-axis to highlight the difference in shape. The electron energy is measured in units of the ponderomotive potential at the peak intensity at which the spectrum is calculated or measured. 
In Fig. 2, the PES of potassium and sodium excited by $3.2 \mu \mathrm{m}$ radiation are compared. In the experiment, an atomic beam produced by a thermal source is directed through the focus of an electrostatic parabolic mirror, where it is crossed by the midIR laser beam. The mirror collects photoelectrons emitted over a $2 \pi$ steradian solid angle, and the photoelectron energy is analyzed by time-of-flight. Using $f / 4$ optics, the mid-IR beam may be focused to a waist diameter of $40 \pm 5 \mu \mathrm{m}$, to achieve a maximum intensity of $3 \mathrm{TW} / \mathrm{cm}^{2}$. The cross sections are not measured in the experiment, due to uncertainties in the beam densities and focal volumes, and the experimental curves are scaled to overlap in order to show the differences in the energy dependence.

The theoretical curves are obtained by a numerical integration of the Schrodinger equation using semi-empirically derived $l$-dependent pseudopotentials for the ion cores [12], and are discussed elsewhere in this volume [6]. The interesting difference between the potentials lies in an attractive well in the $d$ potential of the potassium core, while the sodium is nearly hydrogenic. The $s$ and $p$ potentials of both atoms are more repulsive than the analogous hydrogen potentials and for $l>2$, the potential is dominated by the centrifugal term. The attractive well has a dramatic effect on the shapes of the PES. In both the theory and experiment, the electron yield in sodium falls precipitously above $2 U_{p}$, while in potassium it falls off much more slowly. The interpretation of this is that the attractive well greatly enhances the probability that an electron returning to the core will elastically scatter and contribute to the high-energy part of the spectrum. This is borne out in the theory by the fact that when the $d$ potential in potassium is replaced with that of the hydrogen atom [Fig. 2(a)], the highenergy electron yield drops, and the PES resembles that of sodium.

\section{HIGH HARMONICS AND ATTOSECOND PULSES}

The possibility that the harmonic series produced in HHG might yield very short pulses was suggested [13] soon after HHG was first observed. Since HHG is a high order nonlinear process, it is to be expected that individual harmonics might be shorter than the driving pulse, but in addition, the pulse width might be further reduced if many harmonics could be coherently combined. The effectiveness of this of course depends on whether the harmonics are in phase, but it appears that theoretically there are circumstances in which this is the case. Antoine et al [8] proposed a mechanism in which macroscopic phase matching could select only in-phase contributions to harmonics in the plateau region and create a terahertz train of subfemtosecond pulses. Schafer and Kulander [9] calculated that harmonics at the high energy end of the plateau have spectral phase chirps and relative phases that would permit their compression and combination to form a single subfemtosecond pulse. Evaluating and exploiting mechanisms such as these requires an experimental characterization of the coherence properties of the harmonics. Since this is difficult at the short wavelengths produced in HHG experiments driven by near-infrared sources, it seems useful to study these properties in the visible and UV by using a driving source in the midinfrared. 
Linearly polarized mid-IR light is focused by $f / 4$ optics near the exit aperture of an alkali oven. There is no collimation of the atomic beam, and the width of the $\cos ^{3} \theta$ density profile where it intersects the laser beam is $\sim 1 \mathrm{~mm}$. The atomic density is varied with the oven temperature over a range of 0.1-2 torr. The mid-IR beam is focused to $40 \pm 5 \mu \mathrm{m}$, which implies a confocal parameter of $\sim 3 \mathrm{~mm}$. The interaction region is imaged with unit magnification onto the entrance slit of a 0.18 meter flat field air monochromator which has a gated, intensified CCD camera at its image plane. The collection system's absolute efficiency is calibrated at the third and fifth harmonics by comparison with a calibrated photodiode, and the relative throughput at other wavelengths determined from the emission spectrum of a mercury discharge lamp.

Fig. 3 shows a sample spectrum of Rubidium irradiated by a $3.6 \mu \mathrm{m}, 1.9$ psec pulse. The distribution covers 9 orders of magnitude in signal, and is not adjusted for the spectral response of the collection system. The peak intensity of $2 \mathrm{TW} / \mathrm{cm}^{2}$ is roughly twice the saturation intensity of $1.1(4) \mathrm{TW} / \mathrm{cm}^{2}$, which was determined by the intensity dependence of the total photoionization and harmonic yield. There is a clear odd-harmonic series extending to the $19^{\text {th }}$ harmonic $(190 \mathrm{~nm})$ which is the shortwavelength cutoff of our collection system. The $3 U_{p}+I_{p}$ harmonic cutoff [11] would occur at the $23^{\text {rd }}$ harmonic under these conditions.

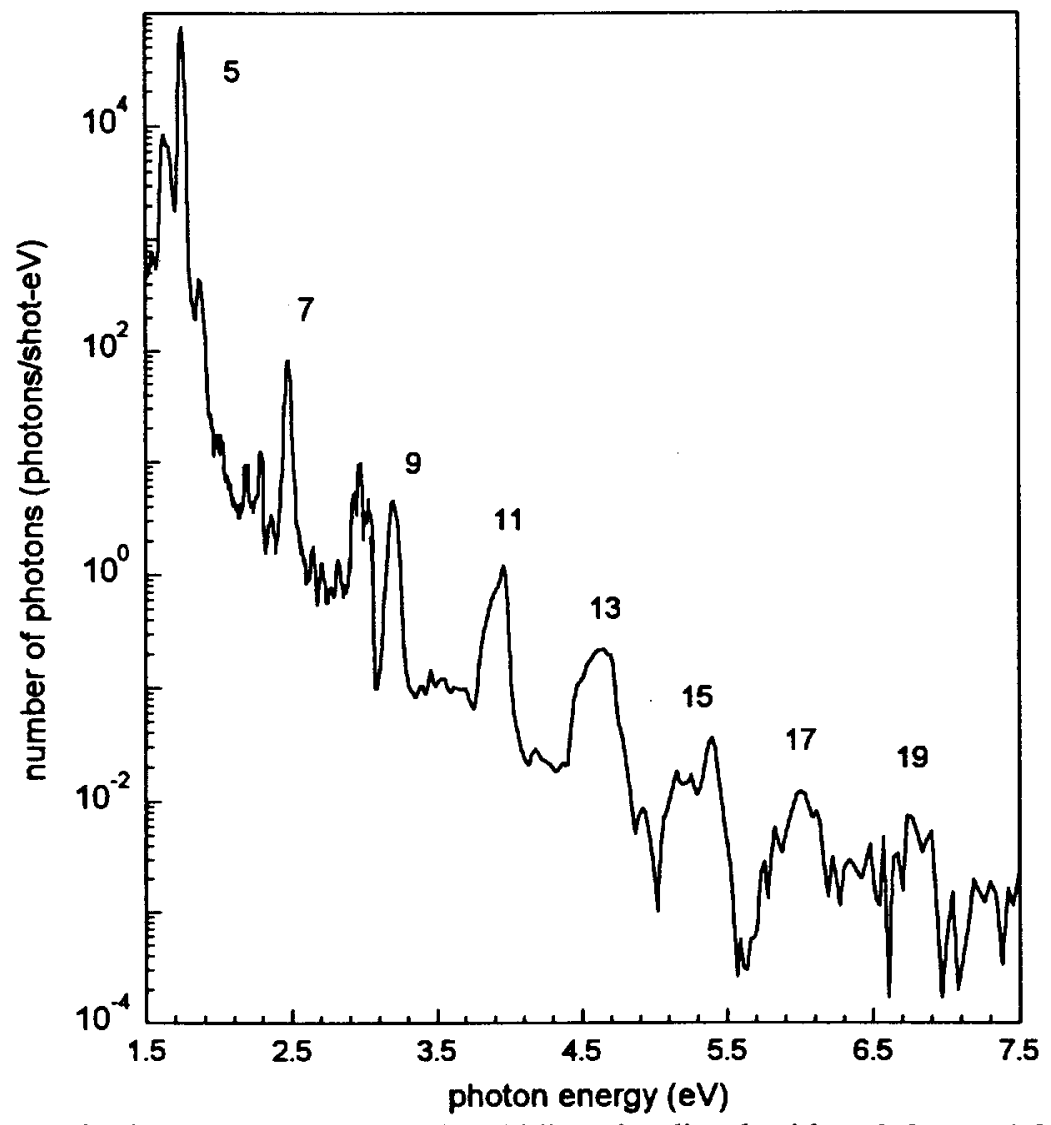

FIGURE 3. The harmonic spectrum of Rubidium irradiated with a $3.6 \mu \mathrm{m}, 1.9 \mathrm{psec}$ pulse (peak intensity $2 \mathrm{TW} / \mathrm{cm}^{2}$ ). Unlabeled lines are due to atomic fluorescence. The spectrum is not corrected for the spectral response of the collection system. 

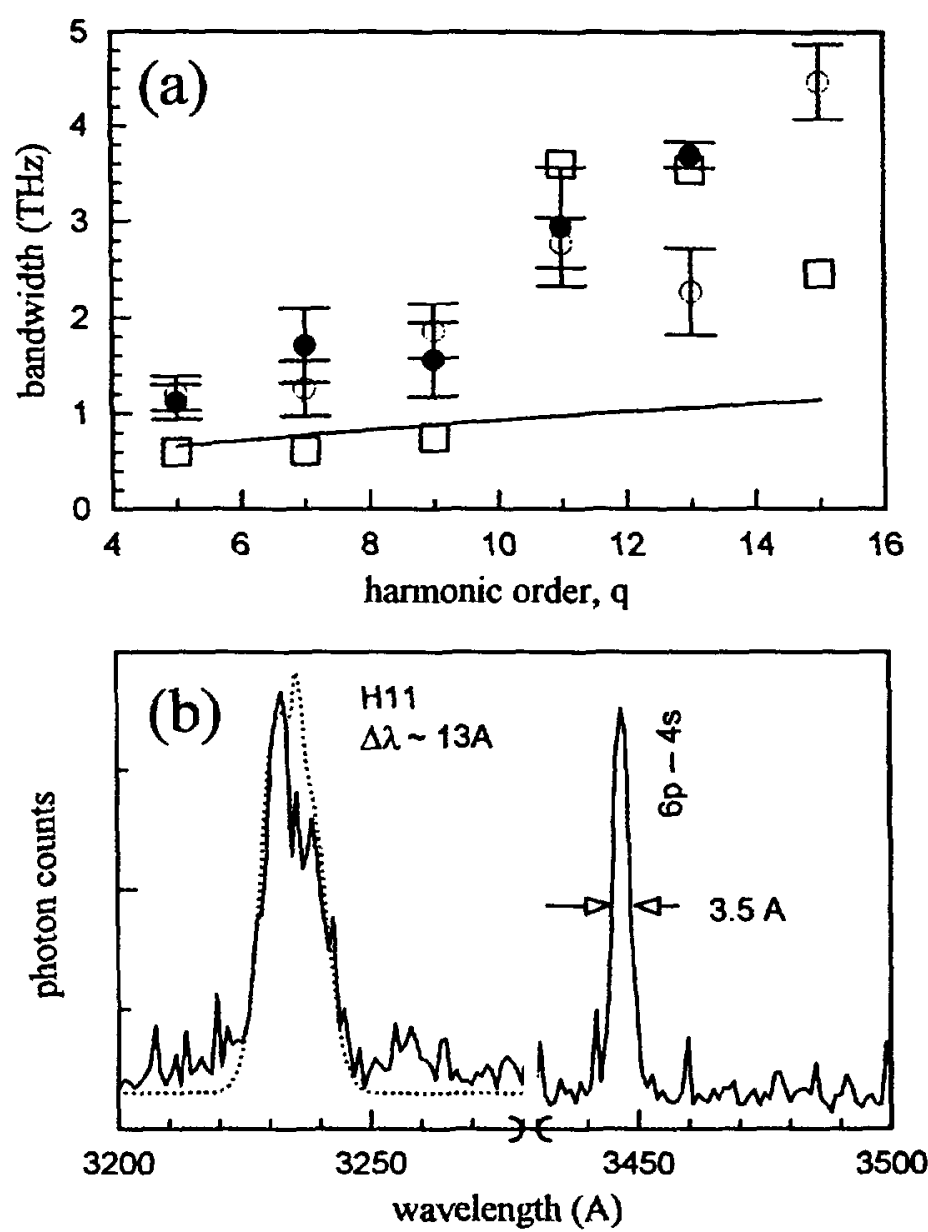

FIGURE 4. (a) Spectral linewidths of harmonics for $\mathrm{Rb}$ excited by $3.2 \mu \mathrm{m}$ radiation (open circles) and $\mathrm{K}$ in a $3.6 \mu \mathrm{m}$ field (filled circles). The open squares are the calculated linewidths of $\mathrm{K}$ at $3.6 \mu \mathrm{m}$. The solid line represents the line width associated with a purely perturbative broadening proportional to $\sqrt{q}$. (b) A typical measured (solid line) and calculated (dotted line) harmonic lineshape in $\mathrm{K}$. The instrumental resolution is shown by the nearby $6 p$ to $4 \mathrm{~s}$ fluorescence line.

We observed that at low atomic densities, the harmonic yield scaled with the square of the density, which implies macroscopic coherence. Near field images confirm that the emission is confined to within a beam waist of the interaction volume, and the harmonic yield shows a weak dependence on the position of the focal spot relative to the atomic distribution, suggesting that we are close to the optimal geometry of a loosely focused beam in which the confocal parameter is approximately equal to the length of the medium [14]. We find that, depending on the harmonic order, rubidium produces harmonics more efficiently than potassium by a factor of $3-6$, for the same atomic number density.

The lineshape of a high order harmonic (H11) produced in $\mathrm{K}$ is shown in Fig. 4(b). The bandwidth is nearly 4 times the instrumental bandwidth, which is shown by a nearby fluorescence line. The bandwidth as a function of harmonic order is shown in Fig. 4(a), for both $\mathrm{Rb}$ and $\mathrm{K}$, at 3.2 and $3.6 \mu \mathrm{m}$ respectively, near the saturation 
intensity. For comparison, we also show the bandwidth that one would expect in the perturbative limit, i.e. if the harmonics were transform-limited and shortened in time relative to the driving pulse by a factor of $\sqrt{q}$, where $q$ is the harmonic order. The low harmonic orders show a small increase in bandwidth, but a larger increase occurs near H11, which coincides with the atomic ionization threshold. The high harmonics show a marked deviation from the perturbative result and suggest that significant temporal compression of the pulse is in principle possible. The theoretical values for $\mathbf{K}$ (open squares) are obtained by numerically calculating the intensity-dependent individual atomic dipole response in the single active electron approximation, and using this as the source term in a solution of the macroscopic electromagnetic wave equation in the paraxial and slowly-varying envelope approximations [15].

It is interesting to ask whether the atomic structure of the alkalis plays as large a role in harmonic production as it does in the photoionization spectra. In fact, the stronger coupling of low-lying excited states (in comparison to the rare gases) does appear to enhance the production of harmonics in the plateau. This is examined theoretically in another paper in this volume [6].

The experimental and theoretical total harmonic yields as a function of harmonic order are shown in Fig. 5. The shape of the yield curve and the relative efficiency of the two atoms are well reproduced. The Z-dependent efficiency is a single-atom effect, and is consistent with harmonic [14] and electron [5] distributions in rare gases. In the absolute photon yields, however, there is a large discrepancy (roughly a factor of 1000 ) between theory and experiment. Our calculations indicate that imperfections in the transverse mode of the mid-IR beam can dramatically reduce the harmonic yield. Our shot-averaged analysis of the beam spot quality does indicate a deviation from TEM $_{00}$, but not enough to completely explain this difference.

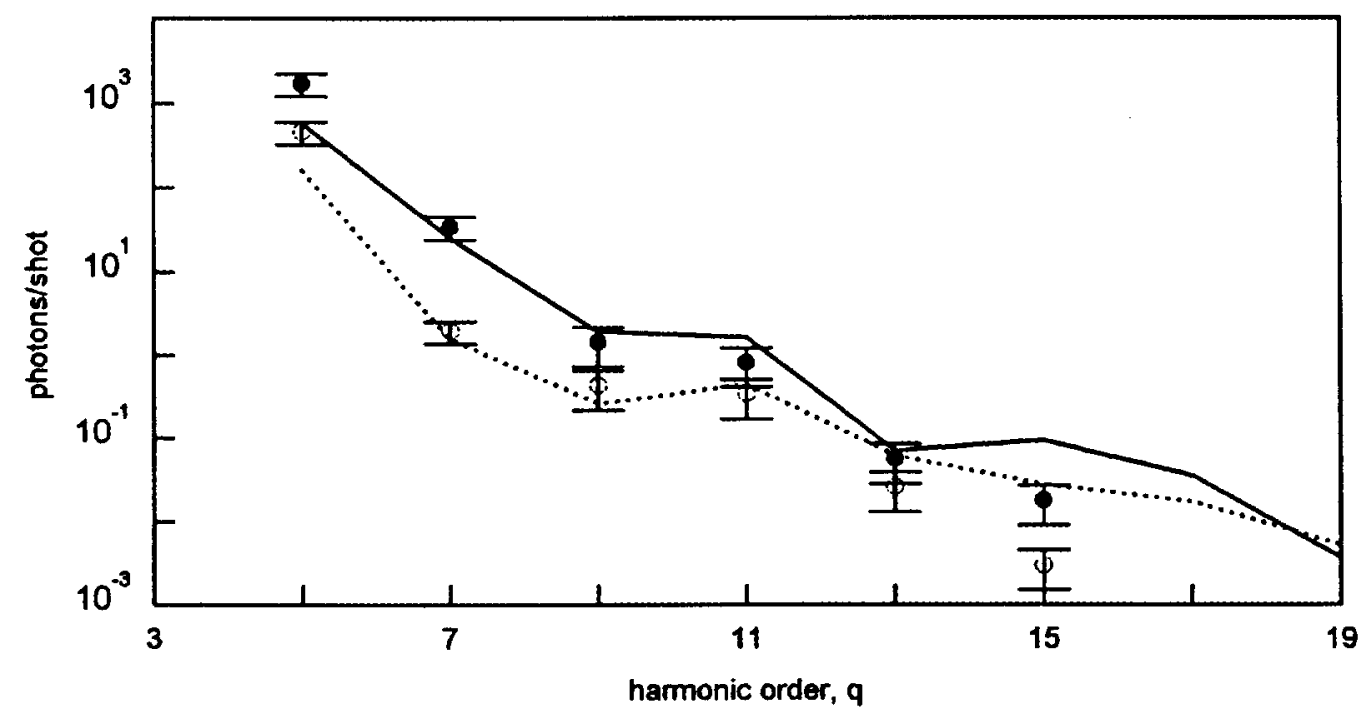

FIGURE 5. Photon yields as a function of harmonic order for $\mathrm{K}$ (open circles) and $\mathrm{Rb}$ (filled circles) in a $3.2 \mu \mathrm{m}, 1.2 \mathrm{TW} / \mathrm{cm}^{2}$ optical field. The calculated yields, scaled by a factor of $10^{-3}$ are shown by the solid $(\mathrm{Rb})$ and dotted $(\mathrm{K})$ lines. 


\section{SUMMARY}

Strong field studies in the mid-IR show a number of interesting contrasts to studies at shorter wavelength. In photoionization, the electron spectra show a greater variation across atomic species. This may be explained by differences in the ion cores, which present very different potentials to returning electrons. The effects of these differences on the photoelectron spectra provide a detailed test of the rescattering model. The alkali atomic structure also plays a role in harmonic generation, where the stronger coupling of low-lying excited states enhances harmonic production at higher orders. We have produced harmonics of a mid-IR field in the visible and UV up to the $19^{\text {th }}$ order, and found that spectral measurements are consistent with theory and with the possibility of pulse compression. The photon yield of harmonics is now sufficiently large to make temporal measurements, as well as measurements which yield complete amplitude and phase information, such as frequency-resolved optical gating [16].

\section{ACKNOWLEDGEMENTS}

The experiments were carried out at Brookhaven National Laboratory under contract No. DE-AC02-98CH10886 with the U.S. Department of Energy and supported by its Division of Chemical Sciences, Office of Basic Energy Sciences and BNL/LDRD No. 99-56. P. A. acknowledge travel support from NATO under Contract No. SA.5-205(RG910678). K.J.S. and M.B.G. acknowledge support from the Louisiana State Board of Regents through Grant No. LEQSF96-99-RD-A-14, from the National Science Foundation through Grant No. PHY-9733890, and the Swedish National Science Research Council. This work was performed in part under the auspices of the U.S. Department of Energy by University of California Lawrence Livermore National Laboratory under contract No. W-7405-Eng-48.

\section{REFERENCES}

1. van Linden van den Heuvell, H. B., and Muller, H. G., "Limiting Cases of Excess-Photon Ionization" in Multiphoton Processes-1988, edited by S. J. Smith and P. L. Knight, Cambridge University Press, 1988, pp 25-34.

2. Schafer, K. J. et al., Phys. Rev. Letters 70, 1599-1602 (1993).

3. Corkum, P. B., Phys. Rev. Letters 71, 1994-1997 (1993).

4. Walker, B. et al., Phys Rev. Letters, 77, 5031-5034 (1996).

5. Sheehy, B, et al., Phys. Rev. A 58, 3942-3952 (1998).

6. Schafer, K. J., et al., this volume.

7. Chang, Z.H., et al. IEEE J. Sel. Top. Quant. Elec., 4, 266-270 (1998); Spielmann, C, et al., IEEE J. Sel. Top. Quant. Elec., 4, 249-265 (1998).

8. Antoine, Ph., L'Huillier, A., and Lewenstein, M., Phys. Rev. Letters 77, 1234-1237 (1996).

9. Schafer, K.J., and Kulander, K. C., Phys. Rev. Letters 78, 638-641 (1997).

10. Hong, J., et al., Appl. Opt. 36, 1894-1897 (1997).

11. Krause J. L., Schafer, K. J., and Kulander, K. C., Physical Review Letters 68, 3535-3538 (1992).

12. Stevens, W. J., et al, Can. J. Chem 70,612-630 (1992) 
13. Harris, S. E., Macklin, J. J., and Hansch, T. W., Opt Commun. 100, 487-490 (1993); Farkas, G., and Toth, C., Phys. Letters A 168, 447-450 (1992).

14. L'Huillier, A., et al., J. Nonlinear Opt. Phys. Mater. 4, 647-665 (1995)

15. Gaarde, M. B., et al., Phys. Rev. A 57, 4553-4560 (1998)

16. Trebino, R., et al., Rev. Sci Instrum. 68, 3277-3295 (1977) 